\title{
CT SPECTRUM OF GIANT RETROPERITONEAL LIPOSARCOMAS WITH HISTOPATHOLOGICAL CORRELATION
}

\author{
Shashikumar M. R¹, Rajendra Kumar N. L², C. P. Nanjaraj ${ }^{3}$, Nishanth R. K ${ }^{4}$ Vishwanath Joshi ${ }^{5}$
}

\section{HOW TO CITE THIS ARTICLE:}

Shashikumar M. R, Rajendra Kumar N. L, C. P. Nanjaraj, Nishanth R. K, Vishwanath Joshi. "CT Spectrum of Giant Retroperitoneal Liposarcomas with Histopathological Correlation". Journal of Evolution of Medical and Dental Sciences 2014; Vol. 3, Issue 46, September 22; Page: 11296-11301, DOI: 10.14260/jemds/2014/3474

ABSTRACT: Liposarcoma constitutes an uncommon and locally aggressive malignancy. We performed a retrospective analysis of 4 patients with histologically proven liposarcoma seen at our institution between 2013 and 2014. All patients underwent either complete surgical resection or debulking surgery. Histological analysis revealed the following subtypes: Well differentiated liposarcoma, well differentiated liposarcoma with myxoid dedifferentiation, Dedifferentiated liposarcoma with myxoid and osteosarcomatous elements and Myxoid dedifferentiation in a case of recurrent well differentiated liposarcoma.

KEYWORDS: liposarcoma, myxoid, dedifferentiation.

INTRODUCTION: Liposarcoma is a malignant tumor of mesenchymal origin. The name liposarcoma implies not that the tumor is derived from fat, but merely the bulk of the tumor tissue has differentiated into adipose tissue. After malignant fibrous histiocytoma, liposarcoma is the second most common soft tissue sarcoma encountered in adults. Most patients with liposarcoma present in $5^{\text {th }}$ and $6^{\text {th }}$ decades. Liposarcomas are usually located in the extremities and in the retroperitoneum. Here we report 4 cases of retroperitoneal liposarcomas with correlative histological diagnosis.

\section{CASES:}

CASE 1: Well differentiated Liposarcoma: A 35 years old female patient was referred for CT scan of abdomen with complaints of abdominal distension. CT scan showed a large retroperitoneal fatattenuation mass lesion extending from the sub hepatic region up to the right iliac fossa. (Fig. 1)The lesion was crossing the midline and causing mass effect in the form of displacement of small bowel loops to the left side. The lesion showed minimally enhancing thick internal septations and fibrous strands (Fig. 2). The patient underwent surgical removal of the mass lesion and a diagnosis of well differentiated liposarcoma was made on the histopathology.

CASE 2: Well differentiated liposarcoma with myxoid Dedifferentiation: A 66 years old female patient was referred for progressive abdominal distension. CT scan shows a large retroperitoneal fatattenuation mass lesion predominantly in midline and extending to both flanks. Mass effect noted in the form of displacement of adjacent small bowel loops and left kidney inferiorly (Fig. 3). The left perinephric component of the lesion had predominantly soft-tissue attenuation within a faint background of fat suggestive of myxoid component. (Fig. 4). Histology confirmed the diagnosis of well differentiated liposarcoma with areas of myxoid dedifferentiation.

CASE 3: Liposarcoma with myxoid and osteosarcomatous Dedifferentiation: A 35 year-old male patient presented with abdominal mass. Contrast enhanced axial CT images shows large left sided 
retroperitoneal mass that is predominantly of soft-tissue attenuation with areas of fat attenuation at the periphery (Fig. 5). Few specks of calcifications noted within the lesion. (Fig. 6) The lesion is seen to cross the midline, causing mass effect in the form displacement left kidney infero laterally, pancreas anteriorly and small bowel loops to the right side. (Fig. 7) Histology showed welldifferentiated liposarcoma with myxoid and osteosarcomatous dedifferentiation.

CASE 4: Myxoid dedifferentiation in recurrent well differentiated Liposarcoma: 70 years old male patient with history of de bulking surgery for well differentiated liposarcoma 10 years previously presented with new abdominal mass. Axial CT image shows soft-tissue mass in the pelvis and extending up to the right sub hepatic region suggestive of myxoid deposits within the residual lesion of liposarcoma. (Fig. 8)

DISCUSSION: Liposarcomas are malignant tumours of mesenchymal origin. They represent the most common primary retroperitoneal sarcomas (33\%). They are mostly seen between the ages of 50 and 70 years, and their incidence increases with age. Liposarcomas are generally large with an average diameter of more than $20 \mathrm{~cm}$. They are slow growing tumours. Their exact aetiology is not understood.[1]

Liposarcoma can be subclassified into three groups: (a) well-differentiated liposarcoma with and without dedifferentiated components, (b) myxoid and round cell liposarcoma, and (c) pleomorphic liposarcoma. These subtypes have radiologic, clinical, pathologic, and genetic distinctions. ${ }^{[2]}$

Imaging features on CT are useful in the diagnosis, prediction of subtype and also help in the follow-up of post-operative patients. As the order of malignancy increases among the various histological subtypes, the amount of fat is reduced to a minimum or absent, resulting in attenuation similar to that of muscle.[3]

Among the various subtypes, the well-differentiated type is the most common. Well differentiated liposarcomas are usually round or lobulated, displacing or surrounding normal structures. They frequently contain septa, as well as occasional non adipose, solid-appearing areas. The solid-appearing areas are poorly defined, with no clear demarcation between them and fat. After administration of contrast material, the solid areas usually enhance.[4]

Dedifferentiated liposarcoma could be considered a subgroup of well-differentiated liposarcoma,[5] characterized by a histologic dedifferentiation with a mixture of both welldifferentiated liposarcoma components and transformed non lipogenic areas. (Normally at highgrade of differentiation) About $10 \%$ of well differentiated liposarcomas undergo dedifferentiation as a time-dependent phenomenon.

Dedifferentiated liposarcoma has a predominant non-fatty solid component with a coexisting fatty component without a clear delineation between the two areas. The fatty component reveals fatattenuation on CT. The CT attenuation of the non lipomatous masses are nonspecific, and may appear hypo (myxoid) to isodense on CT and show variable contrast enhancement.[6]

The myxoid variant is more commonly seen in younger age groups, with a "pseudocystic" appearance on CT due its fat and soft tissue component. They have a heterogeneous density, less than that of muscle. Slow and progressive reticular contrast enhancement of the solid components helps to differentiate the lesion from a cyst. Pleomorphic and round cell liposarcomas appear as 


\section{CASE REPORT}

heterogeneous soft tissue masses with necrotic areas undistinguishable from other solid neoplasms.[6]

The presence of calcifications or ossifications within a liposarcoma often indicates osteosarcomatous dedifferentiation and is associated with poor prognosis. CT shows a high sensitivity for assessing calcifications in such cases.[7]

Surgery is the mainstay treatment of both primary and recurrent retroperitoneal liposarcomas. The treatment of choice is the radical surgical resection obtaining a tumour freemargin, which is the most important predictor of local recurrence and survival. The role of radiation and chemotherapy in the management of retroperitoneal sarcoma remains controversial.[8]

CONCLUSIONS: Liposarcomas are histologically diverse soft-tissue sarcomas with a wide range of appearances on imaging. The classification of LPS has been recently modified with advances in the molecular biology of these tumors, and the radiologist should be aware of the major subtypes. Understanding and recognizing the spectrum of radiologic appearances and the pathologic basis of different subtypes improve patient assessment and help optimize clinical management.

\section{REFERENCES:}

1. Rajiah P, Sinha R, Cuevas C, Dubinsky TJ, Bush WH Jr, Kolokythas O. (2011) Imaging of Uncommon retroperitoneal masses. Radiographics. Jul-Aug; 31(4):949-76.

2. Christopher DM, Unni K, Mertens F (2002) Adipocytic tumors WHO classification of tumors.Pathology and genetics: tumors of soft tissue and bone IARC 19-46

3. Kim EY, Kim SJ, Choi D, Lee SJ, Kim SH, Lim HK, Song SY (2008) Recurrence of Retroperitoneal liposarcoma: imaging findings and growth rates at follow-up CT. AJR Am J Roentgenol. 191 (6): 1841-6.

4. Craig WD, Fanburg-Smith JC, Henry LR, Guerrero R, Barton JH. (2009) Fat-containing lesions of the retroperitoneum: radiologic-pathologic correlation. Radiographics. 29 (1): 261-90.

5. H. L. Evans (1979) Liposarcoma. A study of 55 cases with a reassessment of its classification American Journal of Surgical Pathology vol. 3, no. 6, pp. 507-523.

6. Briani C, Cappucci M, Bucciarelli A, Fantini C, Matteoli M, Di Pietropaolo M, Iannicelli E. (2013, Mar. 15) A large calcified dedifferentiated liposarcoma of the retroperitoneum \{Online\}

URL: http://www.eurorad.org/case.php?id=10789.

7. Tateishi U, Hasegawa T, Beppu Y et al (2003) Primary dedifferentiated liposarcoma of the retroperitoneum. Prognostic significance of computed tomography and magnetic resonance imaging features J Comput Assist Tomogr. 27 (5): 799-804.

8. Strauss DC, Hayes AJ and Thomas JM (2011): Retroperitoneal tumors: review of management Ann R Coll Surg Engl 93 (4): 275-80. 


\section{CASE REPORT}

Figures 1 and 2: Coronal and axial contrast enhanced CT scans showing a large retroperitoneal fat-attenuation mass lesion extending from the sub hepatic region upto the right iliac fossa.The lesion is crossing the midline and causing mass effect in the form of displacement of small bowel loops to the left side. The lesion shows minimally enhancing thick internal septations and fibrous strands (black arrow heads).

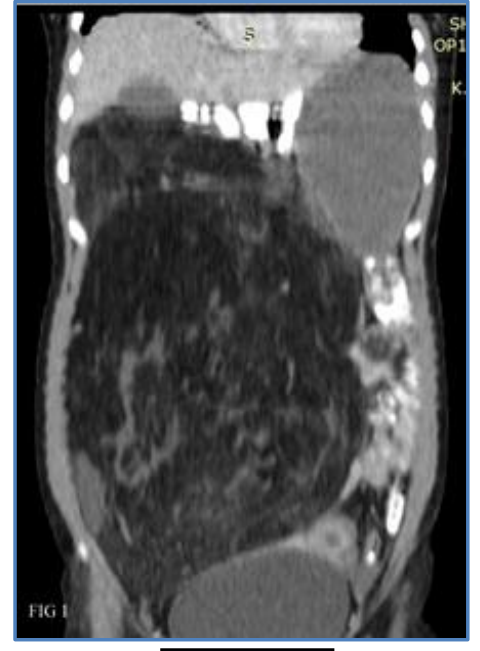

Fig. 1

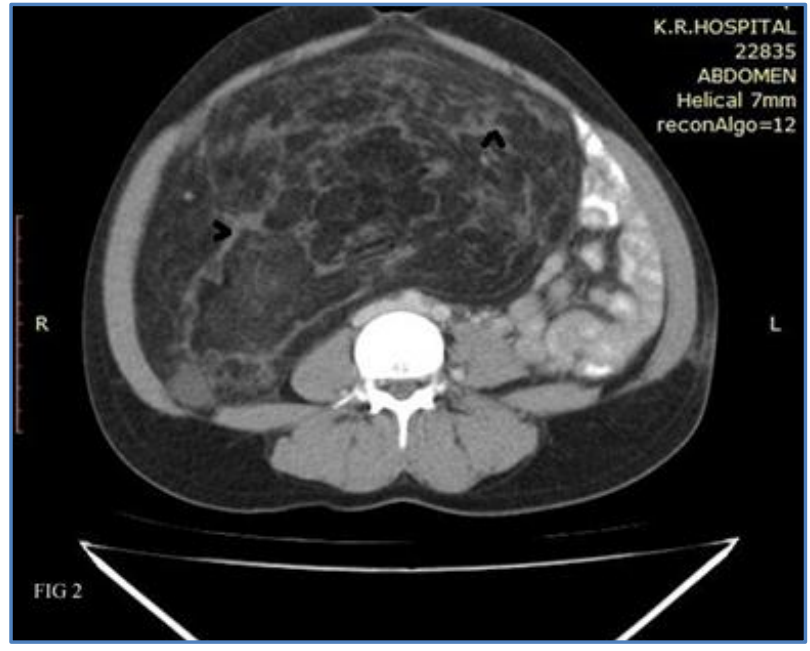

Fig. 2

Figures 3 and 4: Axial contrast enhanced CT scans shows a large retroperitoneal fatattenuation mass lesion predominantly in midline and extending to flanks on either sides. The mass lesion is causing mass effect in the form of displacement of small bowel loops to the either sides. The left perinephric component (black arrow) of the lesion has a predominantly soft-tissue attenuation within a faint background of fat suggestive of myxoid component.

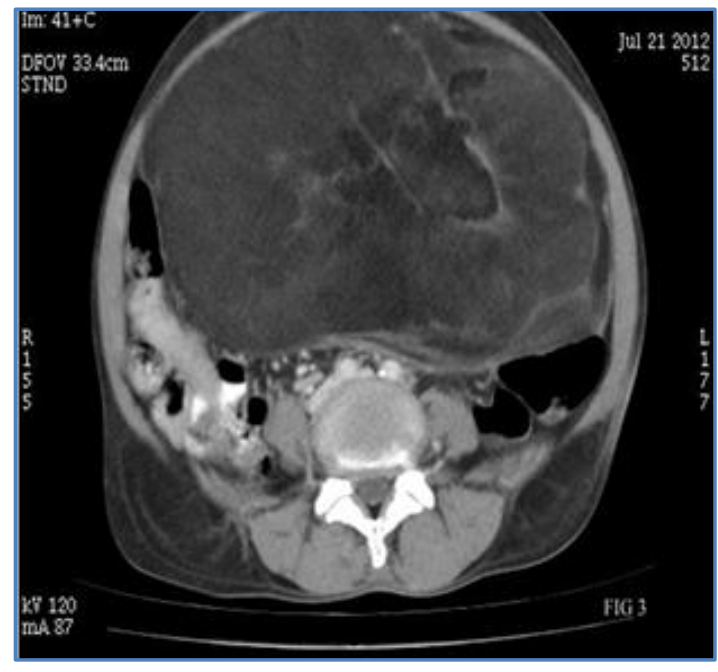

Fig. 3

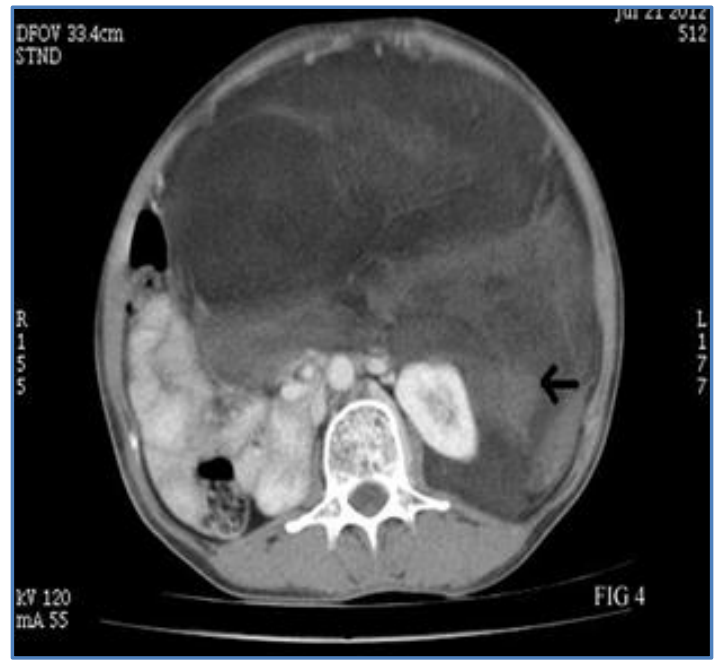

Fig. 4 


\section{CASE REPORT}

Figures 5, 6, 7: Axial contrast enhanced CT images showing a large left -sided retroperitoneal mass that is predominantly of soft-tissue attenuation with areas of fat attenuation at the periphery. Few specks of calcifications noted within the lesion (black arrow). The lesion is seen to cross the midline, causing mass effect in the form displacement of pancreas anteriorly and small bowel loops to the right side.
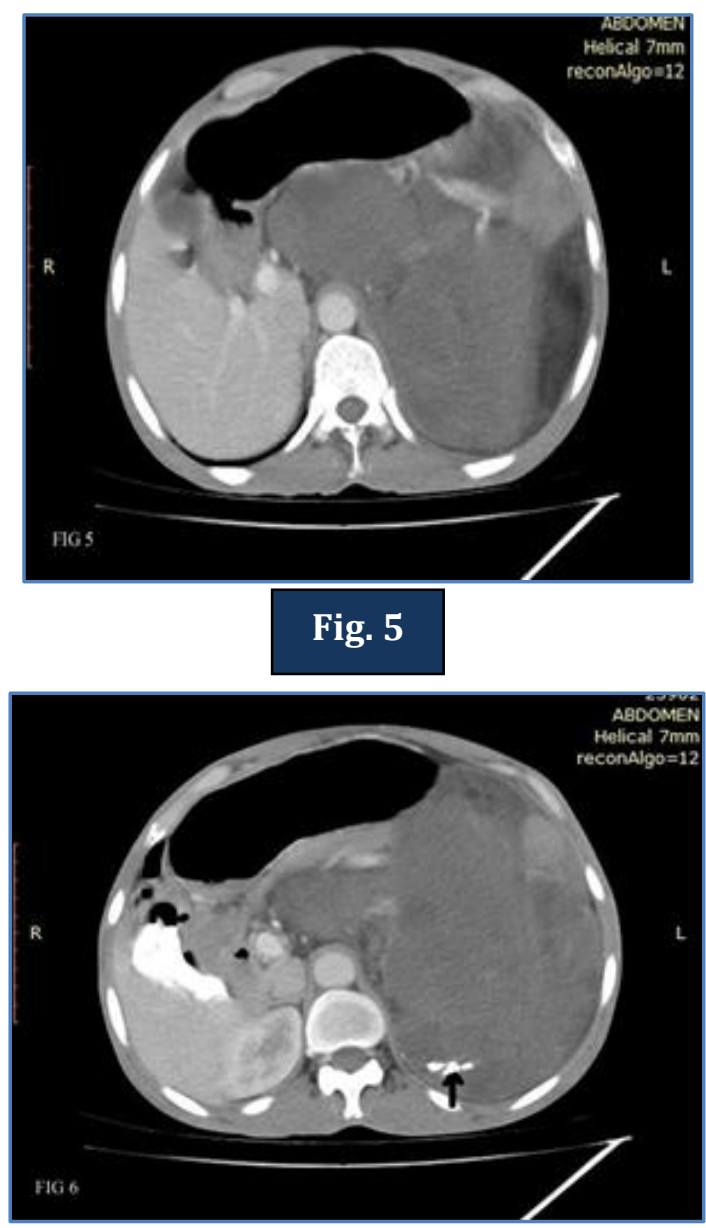

Fig. 6

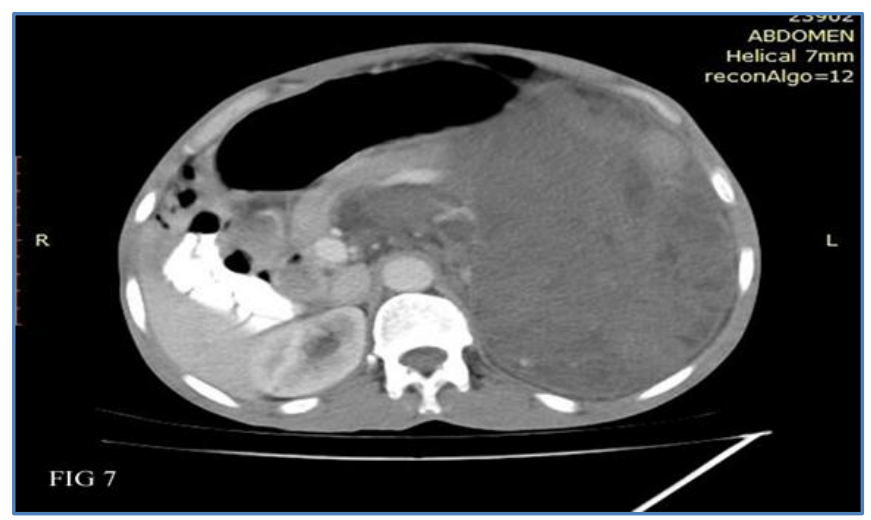

Fig. 7 


\section{CASE REPORT}

Figure 8: Axial CT image showing a soft-tissue mass within the region of residual well differentiated liposarcoma suggestive of myxoid deposits.

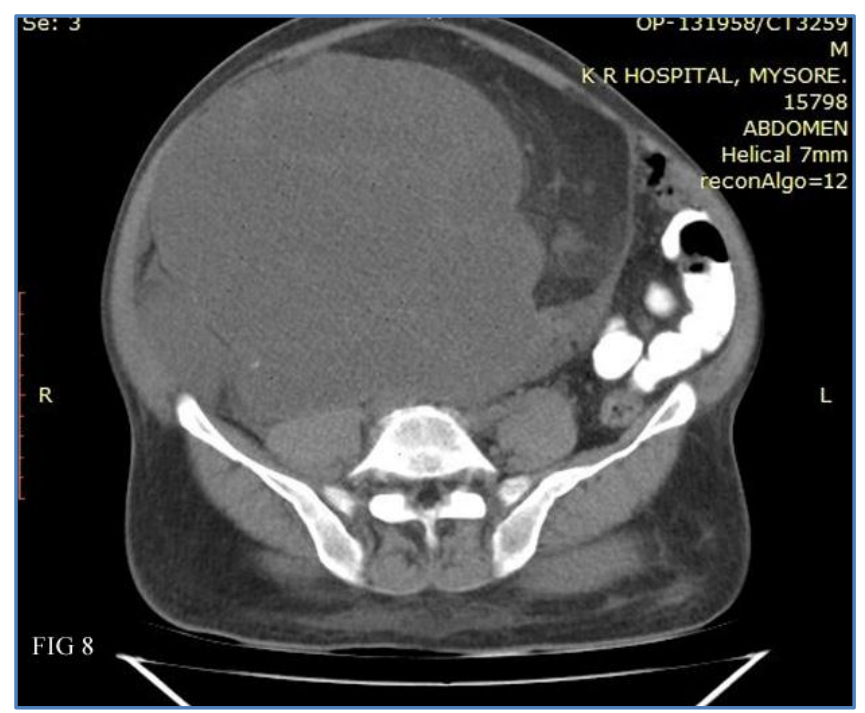

Fig. 8

\section{AUTHORS:}

1. Shashikumar M. R.

2. Rajendra Kumar N. L.

3. C. P. Nanjaraj

4. Nishanth R. K.

5. Vishwanath Joshi

\section{PARTICULARS OF CONTRIBUTORS:}

1. Associate Professor, Department of Radiodiagnosis, Mysore Medical College and Research Institute, Mysore, Karnataka.

2. Associate Professor, Department of Radiodiagnosis, Mysore Medical College and Research Institute, Mysore, Karnataka.

3. Professor, Department of Radiodiagnosis, Mysore Medical College and Research Institute, Mysore, Karnataka.

4. Senior Resident, Department of Radiodiagnosis, Mysore Medical College and Research Institute, Mysore, Karnataka.

5. Post Graduate, Department of Radiodiagnosis, Mysore Medical College and Research Institute, Mysore, Karnataka.

\section{NAME ADDRESS EMAIL ID OF THE CORRESPONDING AUTHOR:}

Dr. Shashikumar M. R, \#241, $1^{\text {st }}$ Stage,

Brindavan Extension,

Mysore-570020.

Email: drmrshashikumar@gmail.com

Date of Submission: 05/09/2014.

Date of Peer Review: 06/09/2014.

Date of Acceptance: 13/09/2014.

Date of Publishing: 22/09/2014. 